05

\title{
Нелинейные эффекты теории тепловой стабилизации технических сверхпроводников
}

\author{
(C) В.P. Романовский \\ Научно-исследовательский центр „Курчатовский институт“, \\ 123182 Москва, Россия \\ e-mail: romanovskii@aol.com
}

(Поступило в Редакцию 27 октября 2016 г.)

Исследована устойчивость диссипативных состояний технического сверхпроводника на основе $\mathrm{Bi}_{2} \mathrm{Sr}_{2} \mathrm{CaCu}_{2} \mathrm{O}_{8}$, охлаждаемого жидким гелием или водородом, с непрерывно нарастающей вольт-амперной характеристикой, описанной степенным уравнением. Показано, что при интенсивных условиях охлаждения могут существовать особые условия тепловой стабилизации технических сверхпроводников, если учитывать нелинейные температурные зависимости критической плотности тока и удельного электрического сопротивления стабилизирующей матрицы. Во-первых, могут отсутствовать минимальные токи существования и распространения нормальной зоны. Во-вторых, интенсивное охлаждение технического сверхпроводника значительно увеличивает диапазон устойчивых токов в закритической области токов. В-третьих, нарастание температуры технического сверхпроводника при необратимом распространении тепловой неустойчивости может происходить в условиях, близких к адиабатическим, несмотря на его охлаждение жидким хладагентом. Данные эффекты необходимо учитывать при определении условий пережога технического сверхпроводника. Выполненные численные эксперименты сопоставлены с результатами, следующими из теории тепловой стабилизации комбинированных сверхпроводников, предполагающей линейную зависимость плотности критического тока сверхпроводника от температуры и скачкообразный переход из сверхпроводящего состояния в нормальное.

DOI: $10.21883 /$ JTF.2017.11.45124.2084

\section{Введение}

В основе явлений, происходящих в технических низкои высокотемпературных сверхпроводниках, изготовленных в виде композитных проводников, лежат коллективные теплоэлектродинамические процессы, которые могут быть описаны в рамках макроскопического приближения. На его основе разработаны теории устойчивости сверхпроводящего состояния при действии возмущений различной природы [1-3], в частности теория тепловой стабилизации комбинированных сверхпроводников, позволяющая определить условия разрушения их сверхпроводящих свойств тепловыми неустойчивостями. Ее основные положения были сформулированы в предположении линейной зависимости критической плотности тока сверхпроводника от температуры для идеальной вольт-амперной характеристики (BAX) сверхпроводника. В этом случае деление тока между сверхпроводником и стабилизирующей матрицей начинается тогда, когда температура технического сверхпроводника превышает так называемую температуру резистивного перехода. В рамках данного приближения процесс деления тока между сверхпроводником и матрицей зависит только лишь от уменьшения с температурой критической плотности тока сверхпроводника. В то же время очевидно, что реальный процесс деления тока должен зависеть от степени нелинейности ВАХ сверхпроводника, сопротивления стабилизирующей матрицы. Однако закономерности развития тепловых процессов в технических сверхпроводниках, определяющих специфику их развития с учетом реального механизма деления тока, изучены не полностью. В связи с этим в настоящей работе обсуждаются возможные сценарии теплового разрушения сверхпроводящих свойств комбинированного проводника, изготовленного из высокотемпературного сверхпроводника с непрерывной ВАХ, принимая во внимание нелинейные зависимости критической плотности тока и сопротивления матрицы от температуры.

\section{Используемые модели}

Рассмотрим технический сверхпроводник с площадью поперечного сечения $S$, находящийся в постоянном магнитном поле $B$. Будем считать, что по нему течет постоянный ток с плотностью $J$ в направлении продольной оси $X$, и он охлаждается жидким хладагентом с периметром охлаждения $p$. Предположим также, что температуpa $T$ и напряженность электрического поля $E$ постоянны по сечению технического сверхпроводника; тепловой поток $q(T)$ с его поверхности в хладагент характеризуется скачкообразным переходом от пузырькового режима кипения к пленочному [1] после ее перегрева на величину $\Delta T_{\text {cr }}$; между сверхпроводником и матрицей имеются идеальные тепловой и электрический контакты; объемная доля сверхпроводника равна $\eta$; его ВАХ описывается степенным уравнением $E(J)=E_{c}\left(J / J_{c}\right)^{n}$, где $J_{c}$ - критическая плотность тока сверхпроводника, определенная на основе использования априори задан- 
ного значения напряженности электрического поля $E_{c}$, $n$ - параметр нарастания ВАХ.

В рамках данных предположений, упрощающих проводимый анализ, напряженность электрического поля для заданных значений плотности тока и температуры удовлетворяет следующей системе уравнений:

$$
\begin{gathered}
E=E_{c}\left[J_{s c} / J_{c}(T, B)\right]^{n}=J_{m} \rho_{m}(T, B), \\
J=\eta J_{s c}+(1-\eta) J_{m},
\end{gathered}
$$

где $J_{s c}$ и $J_{m}-$ плотности токов, протекающих в сверхпроводящей части технического сверхпроводника и в стабилизирующей матрице соответственно; $\rho_{m}$ удельное электрическое сопротивление матрицы.

Исследуем также тепловые режимы технического сверхпроводника в рамках модели скачкообразного перехода из сверхпроводящего состояния в нормальное (идеальная ВАХ). Если использовать линейную аппроксимацию температурной зависимости критической плотности тока сверхпроводника вида $J_{c}=J_{c 0}\left(T_{c B}-T\right) /\left(T_{c B}-T_{0}\right)$, то напряженность электрического поля, индуцированная в техническом сверхпроводнике, описывается формулой $[1-3]$

$$
E(T)=\frac{J \rho_{m}(T, B)}{1-\eta}\left\{\begin{array}{l}
1, T>T_{c B} \\
\left(T-T_{c s}\right) /\left(T_{c B}-T_{c s}\right), \\
T_{c s} \leq T \leq T_{c B} \\
0, T<T_{c s}=T_{c B}-\left(T_{c B}-T_{0}\right) J /\left(\eta J_{c 0}\right) .
\end{array}\right.
$$

Здесь $J_{c 0}$ и $T_{c B}-$ критическая плотность тока сверхпроводника и его критическая температура при температуре хладагента $T_{0}$ для заданного внешнего магнитного поля $B ; T_{c s}$ - температура резистивного перехода.

Выполним анализ возможных условий тепловой стабилизации для технического сверхпроводника на основе $\mathrm{Bi}_{2} \mathrm{Sr}_{2} \mathrm{CaCu}_{2} \mathrm{O}_{8}$ в серебряной матрице $\left(\mathrm{Ag} / \mathrm{Bi}_{2} \mathrm{Sr}_{2} \mathrm{CaCu}_{2} \mathrm{O}_{8}\right)$ и находящегося в магнитном поле с индукцией $B=15 \mathrm{~T}$, используя данные, приведенные в [4]. Соответственно представленные ниже результаты были получены при $S=1.23 \cdot 10^{-2} \mathrm{~cm}^{2}, p=0.47 \mathrm{~cm}$, $n=10, \eta=0.263, E_{c}=10^{-6} \mathrm{~V} / \mathrm{cm}$.

В качестве охладителя рассмотрим жидкие гелий и водород. Как и в $[4,5]$, тепловой поток $q(T)$ в данные охладители опишем формулами

$$
q(T)\left[\mathrm{W} / \mathrm{cm}^{2}\right]= \begin{cases}2.15\left(T-T_{0}\right)^{1.5}, & T \leq T_{0}+\Delta T_{\mathrm{cr}} \\ 0.06\left(T-T_{0}\right)^{0.82}, & T>T_{0}+\Delta T_{\mathrm{cr}}\end{cases}
$$

при $T_{0}=4.2 \mathrm{~K}, \Delta T_{\mathrm{cr}}=0.6 \mathrm{~K}$ для жидкого гелия и

$$
q(T)\left[\mathrm{W} / \mathrm{cm}^{2}\right]= \begin{cases}0.66\left(T-T_{0}\right)^{2.6}, & T \leq T_{0}+\Delta T_{\mathrm{cr}}, \\ 0.24\left(T-T_{0}\right)^{1.1}, & T>T_{0}+\Delta T_{\mathrm{cr}}\end{cases}
$$

для жидкого водорода при $T_{0}=20 \mathrm{~K}, \Delta T_{\mathrm{cr}}=3 \mathrm{~K}$.

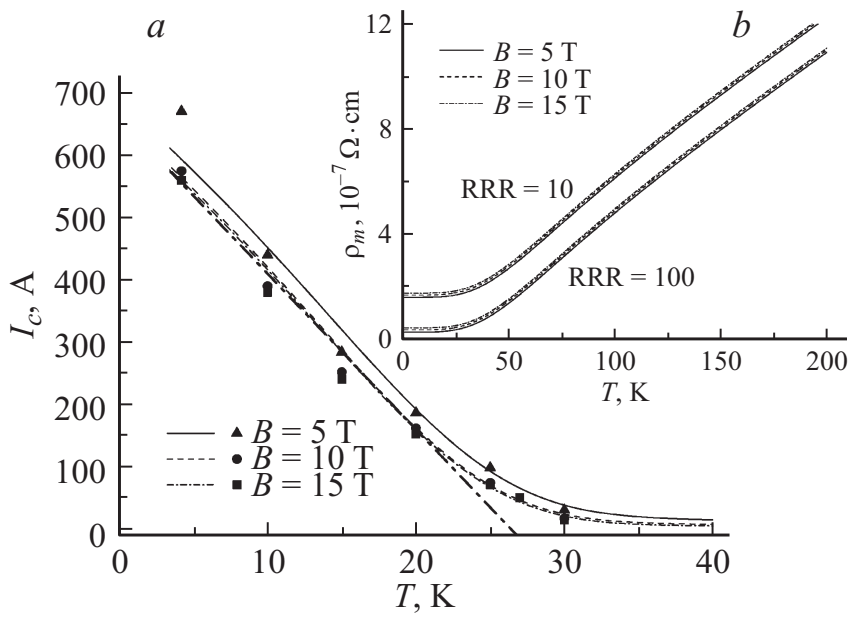

Рис. 1. Зависимости от температуры критического тока $\mathrm{Ag} / \mathrm{Bi}_{2} \mathrm{Sr}_{2} \mathrm{CaCu}_{2} \mathrm{O}_{8}(a)$ и удельного электрического сопротивления серебра $(b):(\boldsymbol{\Lambda}, \bullet, \mathbf{\square})$ - эксперимент, (сплошные, штриховые, штрихпунктирные линии) - расчетные значения.

Критическую плотность тока $\mathrm{Bi}_{2} \mathrm{Sr}_{2} \mathrm{CaCu}_{2} \mathrm{O}_{8}$ будем рассчитывать по формуле, предложенной в [6]

$$
\begin{gathered}
J_{c}(T, B)=J_{0}\left(1-\frac{T}{T_{c}}\right)^{\gamma} \beta_{1}(T, B), \\
\beta_{1}(T, B)=\left[(1-\chi) \frac{B_{0}}{B_{0}+B}+\chi \exp \left(-\frac{\beta B}{B_{c 0} \exp \left(-\alpha T / T_{c}\right)}\right)\right]
\end{gathered}
$$

при $T_{c}=87.1 \mathrm{~K}, \alpha=10.3, \beta=3.3, \gamma=1.73, \chi=0.27$, $B_{c 0}=465 \mathrm{~T}, \quad B_{0}=75 \cdot 10^{-3} \mathrm{~T}, \quad J_{0}=1.1 \cdot 10^{6} \mathrm{~A} / \mathrm{cm}^{2} \quad$ согласно [4]. На рис. 1, $a$ представлены экспериментальные и расчетные зависимости критического тока сверхпроводника $I_{c}=\eta J_{c} S$ от температуры для различных значений индукции внешнего магнитного поля.

Формула (5) также использовалась для оценки соответствующих значений $J_{c 0}$ и $T_{c B}$, необходимых для описания $J_{c}(T, B)$ в линейном приближении (рис. $\left.1, a\right)$. В результате, для рассматриваемого технического сверхпроводника линейная аппроксимация приводит к $J_{c 0}=1.736 \cdot 10^{5} \mathrm{~A} / \mathrm{cm}^{2}, T_{c B}=26 \mathrm{~K}$ при $B=15 \mathrm{~T}$. В этом случае его критический ток $I_{c 0}=\eta J_{c 0} S$ равен $561 \mathrm{~A} \mathrm{при}$ $T_{0}=4.2 \mathrm{~K}$ и $I_{c 0}=154 \mathrm{~A}$ при $T_{0}=20 \mathrm{~K}$.

Для расчета удельного электрического сопротивления серебра использовались результаты [7,8], принимая $\rho_{m}(273 \mathrm{~K})=1.48 \cdot 10^{-6} \Omega \cdot \mathrm{cm}$ при задании $\mathrm{RRR}=$ $=\rho_{m}(273 \mathrm{~K}) / \rho_{m}(4.2 \mathrm{~K})$. На рис. $1, b$ приведены расчетные температурные зависимости удельного электрического сопротивления серебра для двух характерных значений RRR. Они показывают существование в зависимости $\rho_{m}(T)$ двух характерных областей: при $T<20 \mathrm{~K}$ удельное электрическое сопротивление серебра слабо зависит от температуры, а при $T>50 \mathrm{~K}$ оно практически линейно возрастает с температурой. Ниже представлены результаты, полученные при RRR = 10 . 


\section{Особенности диссипативных состояний технических сверхпроводников}

На рис. 2,3 сплошными и штриховыми линиями показаны температурные зависимости джоулевых тепловыделений $G=E J$ при различных значениях транспортного тока. Зависимости, следующие из модели (1), изображены сплошными линиями. Штриховые линии соответствуют тепловыделениям, рассчитанным по модели (2). Штрихпунктирные линии описывают зави-

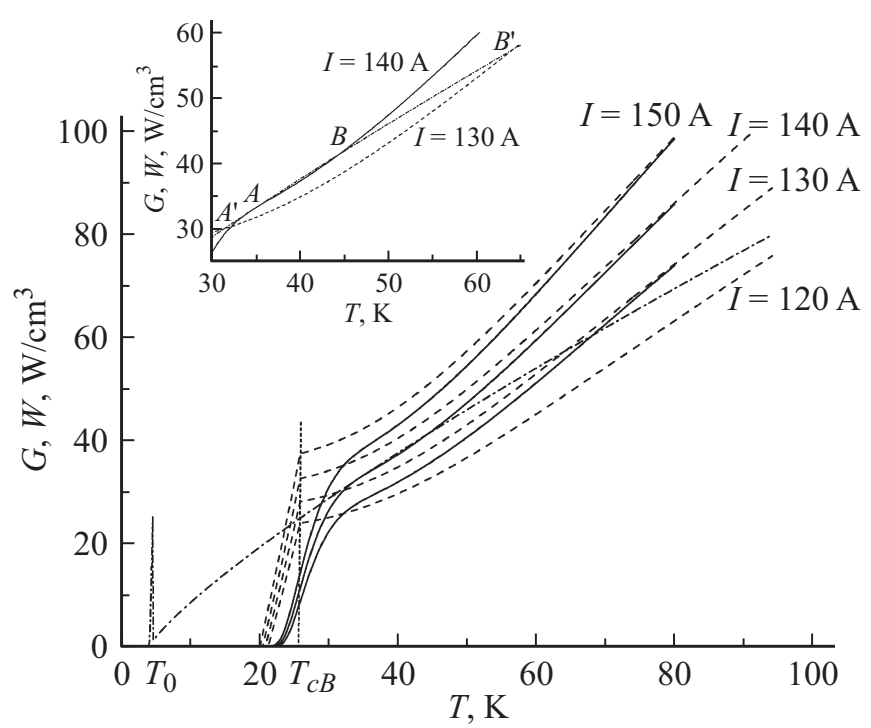

Рис. 2. Температурные зависимости джоулева тепловыделения $G$ и теплового потока в жидкий гелий $W$ при токах, приводящих к неинтенсивным тепловыделениям.

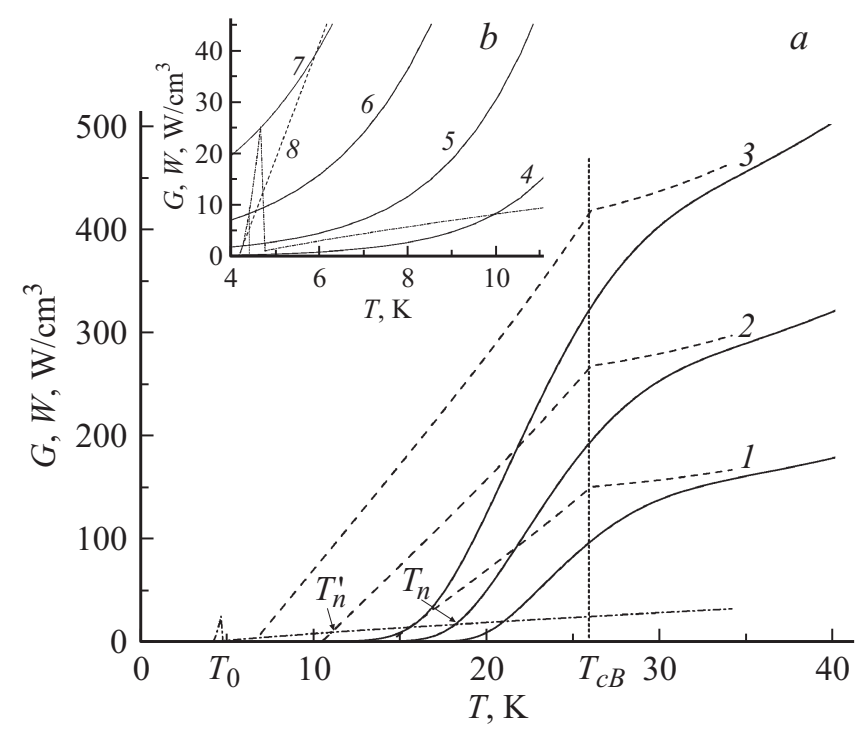

Рис. 3. Температурные зависимости джоулева тепловыделения (сплошные и штриховые линии) и теплового потока в жидкий гелий (штрихпунктирные) при докритических $(a)$ и закритических $(b)$ токах. Модель (1): $1-I=300,2-400,3-500$, $4-700,5-800,6-900,7-1018$ А. Модель (2): $8-$ $I=0.99 I_{c 0}$. симость от температуры мощности теплового потока $W(T)=q(T) p / S$, отводимого в жидкий гелий. Рис. 2 демонстрирует зависимости $G(T)$ и $W(T)$ для токов, у которых джоулевы потери сопоставимы с тепловым потоком в хладагент. Тепловыделения при токах, приводящих к существенному превышению $G(T)$ над значениями $W(T)$ в широком диапазоне изменения температуры $\mathrm{Ag} / \mathrm{Bi}_{2} \mathrm{Sr}_{2} \mathrm{CaCu}_{2} \mathrm{O}_{8}$, и в том числе при токах, которые выше критического тока, приведены на рис. 3. Представленные результаты наглядно демонстрируют влияние нелинейных свойств технических сверхпроводников на устойчивость их диссипативных состояний, которые не только количественно, но и качественно видоизменяют ряд общепринятых положений теории тепловой стабилизации. Обсудим их.

При анализе условий тепловой стабилизации токонесущих элементов сверхпроводящих магнитных систем во внимание необходимо принимать наличие токов, которые определяют особенности возникновения и развития тепловых неустойчивостей. Согласно существующей теории тепловой стабилизации [1-3], диапазон рабочих токов технических сверхпроводников разделяется на три характерные области. Они ограничиваются минимальным током существования нормальной зоны $I_{r}$, минимальным током распространения нормальной зоны $I_{s}$ и критическим током $I_{c 0}$. В каждой из этих областей развитие теплового возмущения происходит по определенному сценарию, так как значения $I_{r}$ и $I_{s}$ зависят от взаимосвязанного изменения с температурой джоулева тепловыделения и теплового потока в хладагент.

Как известно, в диапазоне токов $0<I<I_{r}$ сверхпроводящие свойства технического сверхпроводника при действии произвольного внешнего теплового возмущения не разрушаются, так как после прекращения его действия он возвращается в устойчивое равновесное состояние с температурой, следующей из очевидного равенства: $W(T)=G(T)$ для всех $T<T_{c B}$. В рамках модели идеальной ВАХ равновесная температура технического сверхпроводника в этом случае равна температуре хладагента $T_{0}$, так как $G(T)=0$ при $T<T_{c s}$. У технического сверхпроводника с непрерывной ВАХ соответствующее значение равновесной температуры для заданного тока - это температура $T_{i}$, при которой равенство $W\left(T_{i}\right)=G\left(T_{i}\right)$ выполняется при $G\left(T_{i}\right)>0$. В рассматриваемом случае устойчивое состояние с температурой $T_{i}$ существует при пузырьковом режиме кипения, как это следует из рис. $3, b$. Здесь температура теплового равновесия $T_{i}$ изображена для закритического тока $I=900$ А (кривая 6 ). В целом, значение $T_{i}$, а значит и $G\left(T_{i}\right)$, зависят от величины транспортного тока. Тогда значение $I_{r}$ определяется из условия $W(T) \geq G(T)$ для всех температур, превышающих соответствующее значение температуры теплового равновесия $T_{0}$ или $T_{i}$. В результате, при токе $I_{r}$ должна появиться новая точка устойчивого равновесия. Например, в рамках модели идеальной ВАХ она должна существовать при $T=T_{c B}$, в которой кривые $W(T)$ и $G(T)$ соприкасаются. 
Однако, как следует из рис. 2 , для $\mathrm{Ag} / \mathrm{Bi}_{2} \mathrm{Sr}_{2} \mathrm{CaCu}_{2} \mathrm{O}_{8}$ и с идеальной, и со степенной $\mathrm{BAX}$ данное условие не выполняется в силу интенсивного нарастания c температурой удельного электрического сопротивления стабилизирующей матрицы, происходящее при $T>T_{c B}$. В результате, даже при малых токах саморазогрев $\mathrm{Ag} / \mathrm{Bi}_{2} \mathrm{Sr}_{2} \mathrm{CaCu}_{2} \mathrm{O}_{8}$ будет приводить к превышению мощности джоулева тепловыделения над мощностью теплового потока, отводимого в жидкий гелий, после превышения соответствующего значения температуры. Очевидно, что дестабилизирующая роль температурной зависимости $\rho_{m}$ тем заметнее, чем выше транспортный ток.

Данные особенности нарастания $W(T)$ и $G(T)$ приводят к выводу о том, что у технических сверхпроводников устойчивое равновесное состояние, стабилизирующее неограниченный рост их температуры, может не существовать. Поэтому при закритических тепловых возмущениях (возмущений с энергией, после действия которых происходит необратимый переход сверхпроводника в нормальное состояние) неизбежен пережог сверхпроводника, охлаждаемого жидким гелием, по истечении некоторого времени, зависящего от величины протекающего тока и сопротивления матрицы, вследствие его саморазогрева. Подчеркнем, что данный вывод также справедлив и для низкотемпературных сверхпроводящих композитов.

В общем случае для определения значения критической энергии температурного возмущения необходимо численное решение соответствующего уравнения теплопроводности [3]. Но при действии протяженных возмущений $\left(x_{1} \rightarrow \infty\right)$, когда механизм продольной теплопроводности технического сверхпроводника не оказывает стабилизирующего действия на условия тепловой стабилизации, для нахождения границы устойчивых состояний (в общем случае плотности критической энергии возмущения) можно воспользоваться диаграммами $G(T)-W(T)$. Они позволяют найти температуру неустойчивого равновесия $T_{n}$, после превышении которой сверхпроводящее состояние разрушается [9]. В качестве иллюстрации на рис. 3, $a$ для токового режима 2 приведены соответствующие значения максимально допустимой температуры для бесконечно протяженного температурного возмущения, определенные в рамках обеих моделей ВАХ.

Следует также отметить, что учет нелинейного характера увеличения с температурой удельного электрического сопротивления матрицы может приводить к мультистабильным тепловым состояниям [3]. Данные режимы более детально изображены на вставке к рис. 2. В рассматриваемом случае они возникают при токе 130 А (в диапазоне температур между точками $A^{\prime}$ и $B^{\prime}$ ), если для расчета кривых $G(T)$ и $W(T)$ использовать модель (2), и при токе 140 А (в диапазоне температур между точками $A$ и $B)$, когда используется модель (1). Здесь точки $A$ и $A^{\prime}$ соответствуют устойчивым состояниям, а точки $B$ и $B^{\prime}-$ неустойчивым. Подобные

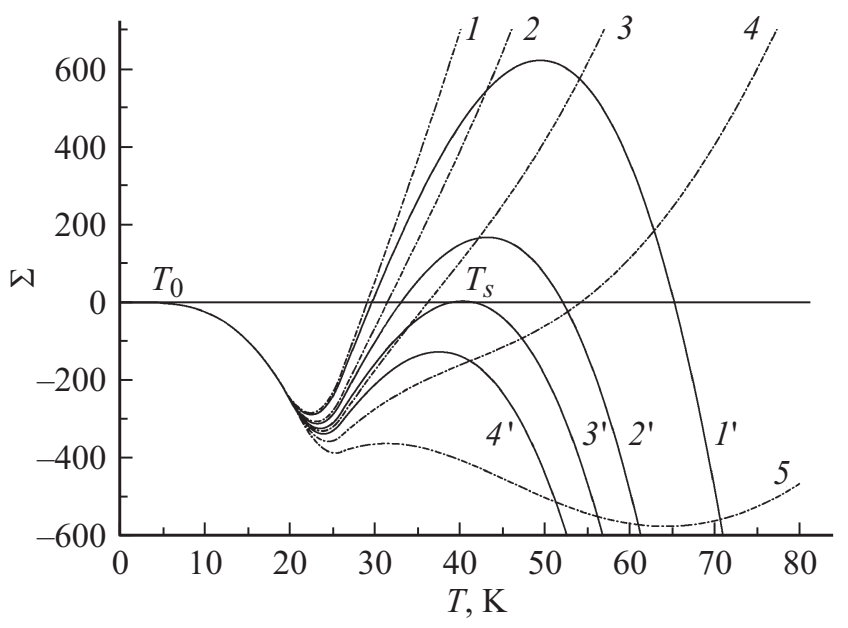

Рис. 4. Диаграммы существования минимального тока распространения нормальной зоны при охлаждении $\mathrm{Ag} / \mathrm{Bi}_{2} \mathrm{Sr}_{2} \mathrm{CaCu}_{2} \mathrm{O}_{8}$ жидким гелием. I, А: $1,1^{\prime}-170 ; 2,2^{\prime}-$ $160 ; 3,4^{\prime}-150 ; 3^{\prime}-155 ; 4-140 ; 5-130$.

состояния также возникают при использовании в качестве хладагента жидкого водорода. Однако обсуждение роли данных мультистабильных состояний в тепловой стабилизации технических сверхпроводников выходит за рамки настоящего исследования.

Минимальный ток распространения нормальной зоны $I_{s}$ определяет токовый режим, когда скорость тепловой неустойчивости по истечению некоторого промежутка времени становится равной нулю [1-3]. Соответственно при $I_{r}<I<I_{s}$ скорости установившегося распространения теплового возмущения вдоль технического сверхпроводника отрицательны. Поэтому при $I_{r}<I<I_{s}$ его сверхпроводящее состояние должно быть устойчивым к произвольным тепловым возмущениям. Значение $I_{s}$ следует из известной теоремы равных площадей $[10]$ и определяется из интегрального равенства

$$
\sum(T)=\int_{T_{0}}^{T}[G(T)-W(T)] \lambda(T) d T=0 \quad \text { при } \quad T=T_{s},
$$

где $T_{s}-$ максимальная равновесная температура сверхпроводника, которая удовлетворяет условиям $\sum\left(T_{s}\right)=0, d \sum\left(T_{s}\right) / d T=0$. Для рассматриваемого режима охлаждения значения $\sum$ как функции температуры при различных токах приведены на рис. 4. Расчеты были выполнены в рамках модели (2). Штрихпунктирные кривые получены в предположении, что удельное электрическое сопротивление матрицы зависит от температуры, а сплошные линии описывают значения $\sum(T)$ при его постоянном значении, равном $\rho_{m}(4.2 \mathrm{~K})$. Видно, что для $\mathrm{Ag} / \mathrm{Bi}_{2} \mathrm{Sr}_{2} \mathrm{CaCu}_{2} \mathrm{O}_{8}$, охлаждаемого жидким гелием, теорема равных площадей не соблюдается, если $\rho_{m}$ непрерывно нарастает с температурой. В то же время при $\rho_{m}=$ const условие $(6)$ выполняется при $I \approx 155 \mathrm{~A}$. Следовательно, при $\rho_{m}=$ const будет существовать и 
минимальный ток существования нормальной зоны $I_{r}$. Поскольку отмеченные особенности существования $I_{s}$ зависят от характера изменения с температурой сопротивления матрицы, то минимальный ток распространения нормальной зоны также отсутствует и для $\mathrm{Ag} / \mathrm{Bi}_{2} \mathrm{Sr}_{2} \mathrm{CaCu}_{2} \mathrm{O}_{8}$ со степенной $\mathrm{BAX}$, как это следует из рис. 2,3 .

Таким образом, учет нелинейных зависимостей $\rho_{m}(T)$ и $q(T)$ может приводить к таким состояниям, когда установившиеся значения скоростей распространения тепловых неустойчивостей будут только положительными и монотонно возрастать с током. Причем согласно результатам, приведенным на рис. 3, в диапазоне температур $T>T_{c B}$ для токов с высокой плотностью выполняется неравенство $G(T) \gg W(T)$. Поэтому после возникновения тепловой неустойчивости температура $\mathrm{Ag} / \mathrm{Bi}_{2} \mathrm{Sr}_{2} \mathrm{CaCu}_{2} \mathrm{O}_{8}$ в наиболее нагретой части даже при охлаждении жидким гелием будет нарастать практически в адиабатических условиях. Данный эффект будет тем заметнее, чем выше ток, который, однако, ограничивается сверху предельно допустимым значением.

Как известно, предельно допустимый ток - это максимальный ток, устойчиво протекающий по техническому сверхпроводнику при отсутствии внешних тепловых возмущений. Его значение в рамках сформулированных моделей (1) и (2) определяется следующим образом. В рамках модели с идеальной ВАХ устойчивые токовые состояния ограничиваются критическим током технического сверхпроводника независимо от условий охлаждения и величины удельного электрического сопротивления матрицы, так как после его превышения произойдет скачкообразный переход из сверхпроводящего состояния в нормальное. В то же время для сверхпроводников с реальной ВАХ условия возникновения токовой неустойчивости (ток срыва $I_{q}$ ) определяются особенностями теплового баланса между выделяемым теплом и тепловым потоком в хладагент даже для композитов на основе низкотемпературных сверхпроводников [9] в силу непрерывного нарастания ВАХ. При использовании в качестве хладагента жидкого гелия токовая неустойчивость в $\mathrm{Ag} / \mathrm{Bi}_{2} \mathrm{Sr}_{2} \mathrm{CaCu}_{2} \mathrm{O}_{8}$ будет возникать после его нагрева до температуры, при которой возникает переход от пузырькового режима кипения жидкого гелия к пленочному, когда $G(T)>W(T)$ для всех $T>T_{0}$. Этот вывод следует из рис. $3, b$. Он также наглядно демонстрирует существование стабильных закритических токовых состояний, даже несмотря на конечный перегрев сверхпроводника. Как следствие, разрушение устойчивого диссипативного состояния $\mathrm{Ag} / \mathrm{Bi}_{2} \mathrm{Sr}_{2} \mathrm{CaCu}_{2} \mathrm{O}_{8}$ происходит при токе $I_{q}=1019 \mathrm{~A}$, в 1.8 раза превышающем критический ток. При этом оказывается, что допустимое увеличение напряженности электрического поля существенно выше априори заданного значения $E_{c}$. Данные состояния возникают в силу интенсивного охлаждения $\mathrm{Ag} / \mathrm{Bi}_{2} \mathrm{Sr}_{2} \mathrm{CaCu}_{2} \mathrm{O}_{8}$ жидким гелием.

Минимальные токи существования и распространения нормальной зоны, отсутствующие при охлаждении

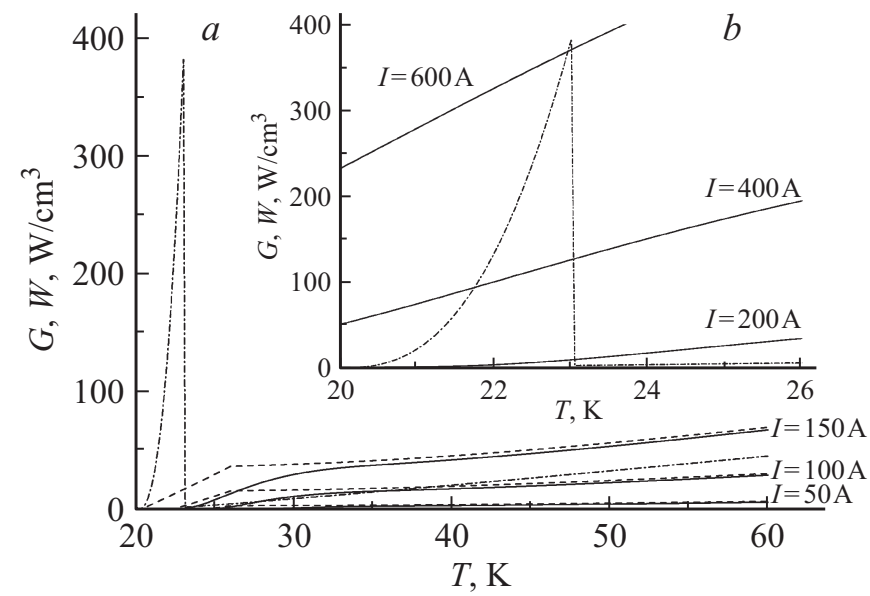

Рис. 5. Температурные зависимости джоулева тепловыделения (сплошные и штриховые линии) и теплового потока в жидкий водород (штрихпунктирные) при докритических $(a)$ и закритических $(b)$ токах.

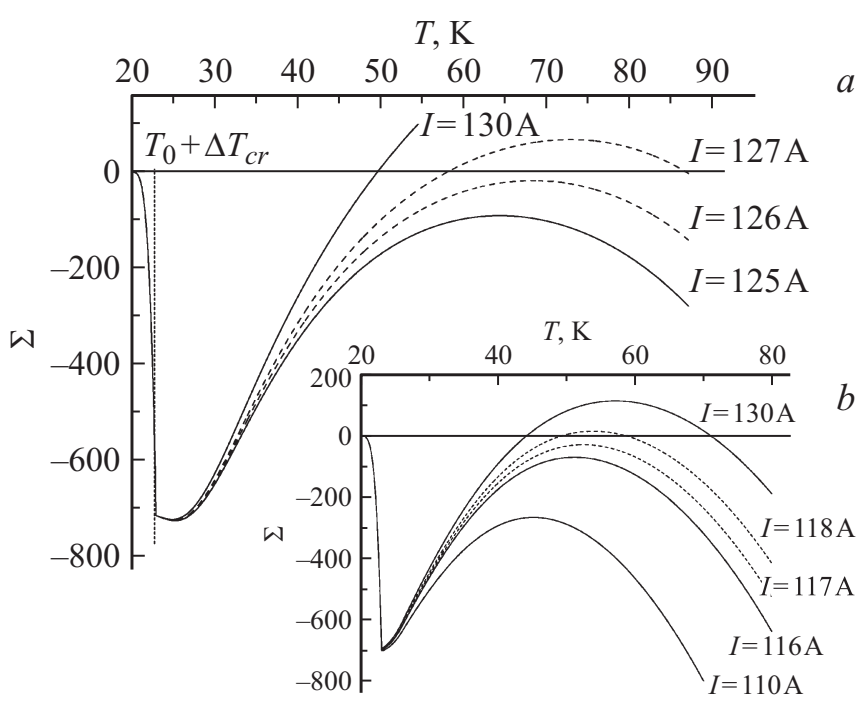

Рис. 6. Расчет минимального тока распространения нормальной зоны при охлаждении $\mathrm{Ag} / \mathrm{Bi}_{2} \mathrm{Sr}_{2} \mathrm{CaCu}_{2} \mathrm{O}_{8}$ жидким водородом: $a$ - модель (1), $b$ - модель (2).

$\mathrm{Ag} / \mathrm{Bi}_{2} \mathrm{Sr}_{2} \mathrm{CaCu}_{2} \mathrm{O}_{8}$ жидким гелием, возникают при его охлаждении жидким водородом (рис. 5,6), т.е. при использовании хладагента, обеспечивающего более интенсивный теплоотвод. Действительно, как следует из рис. 5, $a$, в рамках моделей (1) (сплошные линии) и (2) (штриховые линии) существуют состояния, для которых будет выполняться условие $W \geq G$ при $T>T_{i}$ или $T>T_{0}$ соответственно. Поэтому в этом случае значение $I_{r}$ отлично от нуля, и для всех $0<I<I_{r}$ после прекращения действия какого-либо теплового возмущения $\mathrm{Ag} / \mathrm{Bi}_{2} \mathrm{Sr}_{2} \mathrm{CaCu}_{2} \mathrm{O}_{8}$ будет возвращаться в равновесное состояние, существующее при $T_{i}$ или $T_{0}$ в режиме пузырькового кипения водорода. Кроме этого, результаты расчета значений $\sum(T)$, полученные соглас- 


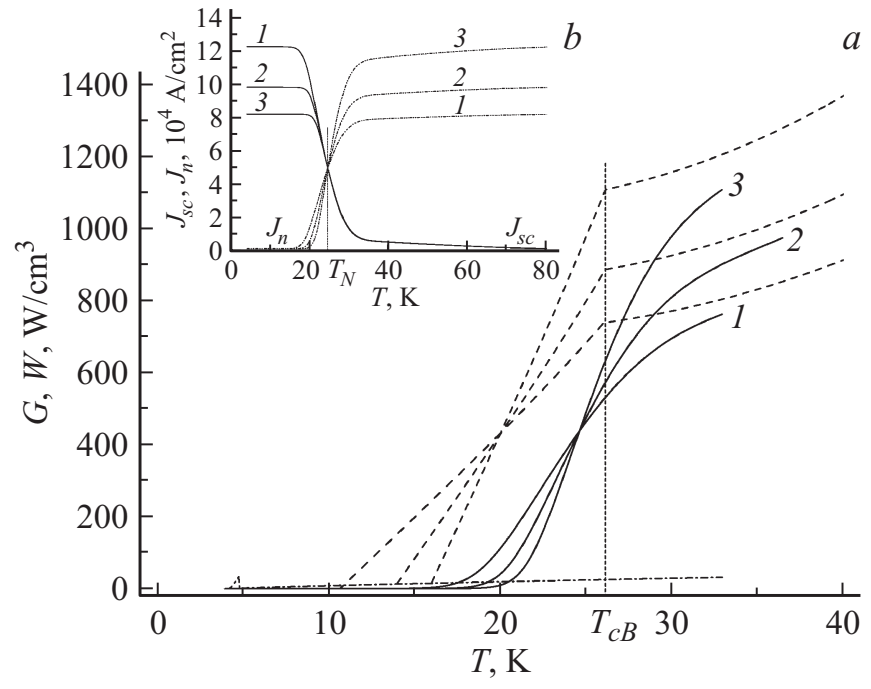

Рис. 7. Влияние коэффициента заполнения на температурные зависимости мощности джоулева тепловыделения при $I=600 \mathrm{~A}(a)$ и изменение с температурой плотности токов в сверхпроводнике $J_{s c}$ и матрице $J_{m}(b): 1-$ $\eta=0.4, I_{c 0}=854 \mathrm{~A} ; 2-\eta=0.5, I_{c 0}=1067 \mathrm{~A} ; 3-\eta=0.6$, $I_{c 0}=1280 \mathrm{~A}$.

но (1), (2) и (6) и представленные на рис. 6, показывают существование тока $I_{s}$ в режиме пленочного кипения хладагента. Соответственно при $I_{r}<I<I_{s}$ установившиеся значения скоростей тепловых возмущений будут отрицательными, так как суммарное тепловыделение в $\mathrm{Ag} / \mathrm{Bi}_{2} \mathrm{Sr}_{2} \mathrm{CaCu}_{2} \mathrm{O}_{8}$ не будет превышать суммарный тепловой поток в хладагент. Абсолютное значение этих скоростей будет максимальным при $I_{r}$, и оно становится равным нулю при $I_{s}$. Нетрудно понять, что отличия в значениях $I_{r}$ и $I_{s}$, рассчитываемых по обеим моделям $\mathrm{BAX}$, зависят от характера изменения с температурой плотности критического тока $\mathrm{Bi}_{2} \mathrm{Sr}_{2} \mathrm{CaCu}_{2} \mathrm{O}_{8}$.

При $I>I_{s}$ и действии закритических возмущений тепловые неустойчивости будут необратимо распространяться вдоль технического сверхпроводника с положительной скоростью, установившееся значение которой будет увеличиваться с увеличением тока, вплоть до предельно допустимого значения. Как и при охлаждении $\mathrm{Ag} / \mathrm{Bi}_{2} \mathrm{Sr}_{2} \mathrm{CaCu}_{2} \mathrm{O}_{8}$ жидким гелием, так и при его охлаждении жидким водородом, ток срыва, равный $I_{q}=607 \mathrm{~A}$, также определяется переходом от режима пузырькового кипения жидкого водорода к пленочному (рис. 5, b). Он выше соответствующего значения критического тока $\mathrm{Ag} / \mathrm{Bi}_{2} \mathrm{Sr}_{2} \mathrm{CaCu}_{2} \mathrm{O}_{8}$ в 3.9 раза, поскольку режим пузырькового кипения жидкого водорода обеспечивает эффективный теплоотвод джоулевых потерь энергии.

Таким образом, условно заданные значения $E_{c}$ и $I_{c}$ не имеют физического смысла как критических параметров технических сверхпроводников при их интенсивном охлаждении жидким гелием и тем более жидким водородом.
Результаты расчетов, представленные на рис. 3,5, приводят к выводу о том, что с увеличением критического тока технического сверхпроводника его пережог становится более вероятным. Данный вывод наглядно подтверждают $G(T)-W(T)$ диаграммы, приведенные на рис. 7, $a$ для $\mathrm{Ag} / \mathrm{Bi}_{2} \mathrm{Sr}_{2} \mathrm{CaCu}_{2} \mathrm{O}_{8}$, охлаждаемого жидким гелием. Они показывают нарастание с температурой мощности джоулевых потерь энергии при варьировании коэффициента заполнения, приводящего к соответствующему изменению критического тока $\mathrm{Ag} / \mathrm{Bi}_{2} \mathrm{Sr}_{2} \mathrm{CaCu}_{2} \mathrm{O}_{8}$. Расчеты были выполнены в рамках обеих моделей $\mathrm{BAX}$ : идеальной (штриховые линии) и степенной (сплошные линии). Видно, что при высоких критических токах $\mathrm{Ag} / \mathrm{Bi}_{2} \mathrm{Sr}_{2} \mathrm{CaCu}_{2} \mathrm{O}_{8}$ теплоотдача в жидкий гелий, изображенная штрихпунктиной линией, в диапазоне температур $T>T_{c B}$ практически не будет оказывать влияние на его тепловое состояние в наиболее нагретой части, так как значения $G(T)$ оказываются существенно выше $W(T)$. При этом отличие тем значительнее, чем больше критический ток. Соответственно возрастает вероятность быстрого пережога технического сверхпроводника.

Следует также отметить существование характерной температуры $T_{N}$, при которой тепловыделение в техническом сверхпроводнике не зависит от коэффициента заполнения. Ее возникновение следует из второго уравнения системы (1), согласно которому соблюдается условие $J_{s c}=J_{m}=J$ как для сверхпроводника с идеальной BAX, так и со степенной. Данный режим показан на рис. 7, $b$ для сверхпроводника со степенной $\mathrm{BAX}$. Для определения значения $T_{N}$ можно воспользоваться первым уравнением системы (1). Температура $T_{N}$ появляется в силу различного влияния коэффициента заполнения на значения плотностей токов в сверхпроводящей части и матрице (рис. 7,b), а значит, и на суммарные тепловыделения в техническом сверхпроводнике. Поскольку $J \sim \eta J_{s c}$ при $T<T_{N}$, то увеличение коэффициента заполнения в диапазоне температур $T<T_{N}$ сопровождается уменьшением джоулевых потерь в силу соответствующего уменьшения $J_{s c}$. И наоборот, при $T>T_{N}$ тепловыделения возрастают с увеличением коэффициента заполнения, так как при этом возрастает ток в стабилизирующей матрице.

Результаты, представленные на рис. 2-7, демонстрируют отличие в выполненных расчетах токовых областей тепловой стабилизации технических сверхпроводников по обеим моделям ВАХ. В их основе прежде всего лежат особенности изменения с температурой дифференциального сопротивления технического сверхпроводника. Действительно, в рамках модели с идеальной ВАХ оно скачком переходит от нулевого значения до бесконечно большого. Соответственно тепловыделения в техническом сверхпроводнике отсутствуют пока транспортный ток меньше критического тока независимо от величины удельного электрического сопротивления матрицы. При $I>I_{c}(T)$ часть транспортного тока, равная $I-I_{c}(T)$, 


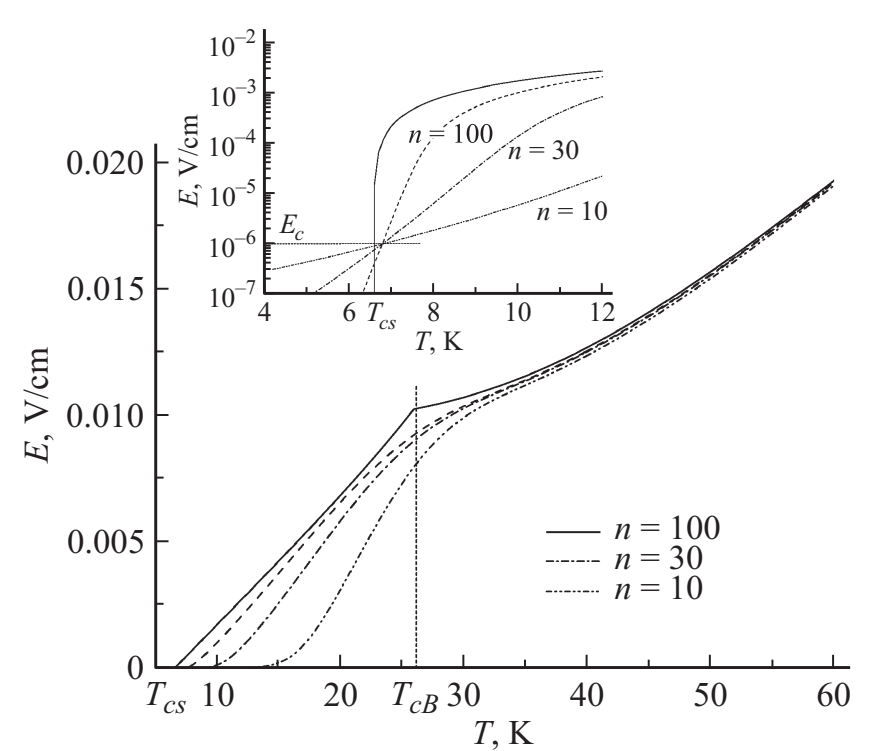

Рис. 8. Напряженность электрического поля в $\mathrm{Ag} / \mathrm{Bi}_{2} \mathrm{Sr}_{2} \mathrm{CaCu}_{2} \mathrm{O}_{8}$ как функция температуры при охлаждении жидким гелием для различных значений параметра нарастания $\operatorname{BAX}(2)$.

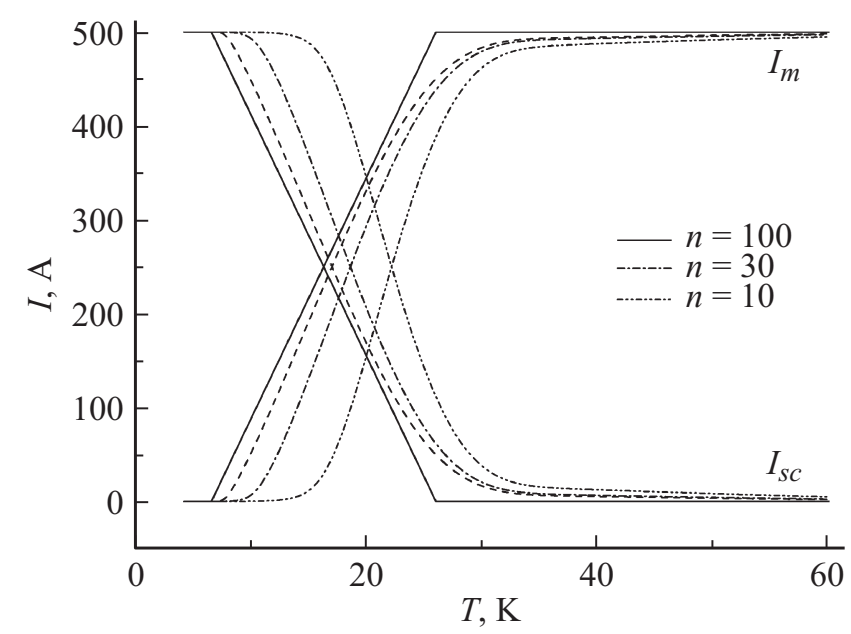

Рис. 9. Влияние параметра нарастания BAX на деление тока между сверхпроводником и матрицей.

начинает перетекать из сверхпроводника в стабилизирующую матрицу. Для сверхпроводящих материалов с реальной ВАХ их дифференциальное сопротивление изменяется непрерывно в зависимости от параметра ее нарастания и температурной зависимости $I_{c}$. Кроме этого, процесс деления тока зависит и от удельного электрического сопротивления стабилизирующей матрицы. Поэтому данные закономерности приводят к характерным тепловыми эффектам, влияющим на вид реальных зависимостей $E(T)$, а значит, и на механизм деления тока. Рис. 8, 9 демонстрируют эти особенности.

На рис. 8 показаны результаты расчета напряженности электрического поля как функции температуры, полученные согласно моделям (1) (сплошная, штрихпунктирная, пунктирная линии) и (2) (пунктир) для $\mathrm{Ag} / \mathrm{Bi}_{2} \mathrm{Sr}_{2} \mathrm{CaCu}_{2} \mathrm{O}_{8}$, охлаждаемого жидким гелием, при докритическом токе $I=500 \mathrm{~A}$ и различных значениях параметра нарастания ВАХ. На вставке к рис. 8 температурные зависимости $E(T)$ изображены более детально в области низких перегревов. На рис. 9 приведены соответствующие кривые, демонстрирующие особенности деления тока между сверхпроводником и матрицей. Видно, что при $T_{0}<T<T_{c B}$ зависимость $E(T)$, рассчитанная в рамках модели (2), практически линейно нарастает с температурой, так как в этом случае $\rho_{m} \sim$ const при $T<20 \mathrm{~K}$ (рис. $\left.1, b\right)$. При $T=T_{c B}$ зависимость $E(T)$ имеет излом, поскольку в этом случае критический ток равен нулю и весь транспортный ток перетекает в матрицу. Поэтому при $T>T_{c B}$ величина напряженности электрического поля изменяется в соответствии с температурной зависимостью его удельного электрического сопротивления. Напряженность электрического поля, рассчитанная в рамках модели (1), как и следовало ожидать, плавно нарастает с температурой в соответствии с непрерывным изменением дифференциального сопротивления сверхпроводника. Но ее величина оказывается меньше напряженности электрического поля, определенной в рамках модели с идеальной ВАХ. Соответственно ток в сверхпроводящей части $I_{s c}$, рассчитанный в рамках модели (1) при температурах выше температуры резистивного перехода $T_{c s}$, всегда выше критического тока $\mathrm{Ag} / \mathrm{Bi}_{2} \mathrm{Sr}_{2} \mathrm{CaCu}_{2} \mathrm{O}_{8}$ (рис. 9). А поэтому соответствующим образом отличаются и токи в стабилизирующей матрице $I_{m}$, рассчитанные в рамках обеих моделей. Эти особенности наблюдаются даже при очень высоких значениях параметра нарастания $\mathrm{BAX}$, например при $n=100$, которому соответствует практически скачкообразный переход сверхпроводника в нормальное состояние. В целом, отличие между моделями в диапазоне температур $T_{c s}<T<T_{c B}$ заметно увеличивается с уменьшением параметра $n$. Как следствие, модель со степенной ВАХ в широком диапазоне изменения температуры технического сверхпроводника $\left(T>T_{c s}\right)$ будет всегда приводить к заниженным значениям напряженности электрического поля, а значит и тепловыделений. В результате, теоретический анализ условий тепловой стабильности сверхпроводников, выполненный в рамках модели (2), неизбежно приведет к завышенным оценкам скорости распространения тепловой неустойчивости. В то же время, поскольку в области высоких температур $\left(T>T_{c B}\right)$ отличия между расчетными моделями уменьшаются, то они будут приводить к близким оценкам условий пережога сверхпроводящих лент.

Следует отметить существование у технических сверхпроводников со степенной ВАХ температуры $T_{s}$, в которой кривые $E(T)$ пересекаются независимо от значения ее параметра нарастания, как показано на вставке к рис. 8. Согласно (1), это возможно в том случае, если $E=E_{c}$. Тогда значение температу- 
ры, при которой выполняется данное условие, следует из решения трансцендентного уравнения $J=$ $=\eta J_{c}\left(T_{s}\right)+(1-\eta) E_{c} / \rho_{m}\left(T_{s}, B\right)$. Температура $T_{s}^{\prime}$, при которой условие $E=E_{c}$ выполняется для сверхпроводника с идеальной ВАХ, определяется из решения уравнения $E_{c}=\frac{J \rho_{m}\left(T_{s}^{\prime}, B\right)}{1-\eta} \frac{T_{s}^{\prime}-T_{c s}}{T_{c B}-T_{c s}}$. Следовательно, при температурах $T>T_{s}$ (для сверхпроводника с идеальной BAX при $T>T_{s}^{\prime}$ ) деление тока между сверхпроводником и стабилизирующей матрицей происходит при закритических значениях напряженности электрического поля даже при незначительных перегревах относительно температуры резистивного перехода. Этот вывод наглядно демонстрирует возможность возникновения в технических сверхпроводниках стабильных закритических состояний.

Для того чтобы понять характер изменения зависимостей $E(T)$, выполним их оценку. При малых перегревах ток в стабилизирующей матрице практически отсутствует (рис. 7,b). Поэтому согласно (1), (5), соблюдается связь

$$
\frac{E}{E_{c}} \cong\left(\frac{J}{\eta J_{0}\left(1-T / T_{c}\right)^{\gamma} \beta_{1}}\right)^{n} .
$$

Прологарифмируем данное равенство. Это приведет к следующему соотношению:

$$
\ln \frac{E}{E_{c}}=n\left[\ln \frac{J}{\eta J_{0} \beta_{1}}-\gamma \ln \left(1-\frac{T}{T_{c}}\right)\right] .
$$

Так как $T / T_{c} \ll 1$, то раскладывая $\ln \left(1-T / T_{c}\right)$ в степенной ряд, получим

$$
\ln \frac{E}{E_{c}}=n\left[\ln \frac{J}{\eta J_{0} \beta_{1}}+\gamma\left(\frac{T}{T_{c}}+\frac{T^{2}}{2 T_{c}^{2}}+\ldots\right)\right] .
$$

Тогда

$$
E(T)=E_{c}\left(\frac{J}{\eta J_{0} \beta_{1}}\right)^{n} \exp \left[n \gamma\left(\frac{T}{T_{c}}+\frac{T^{2}}{2 T_{c}^{2}}+\ldots\right)\right] .
$$

Поскольку $\beta_{1}$ при $T / T_{c} \ll 1$ слабо зависит от температуры (это следует из (5)), то начальный участок зависимости $E(T)$ экспоненциально возрастает с температурой с показателем нарастания, равным $n \gamma T / T_{c}$. Данные выводы подтверждает вставка к рис. 8.

Для оценки $E(T)$ при $T_{0}<T \leq T_{c B}$ учтем, что в этом случае $\rho_{m} \sim$ const. Поэтому из (1) следует равенство $i=$ $=j_{c}(\theta) e^{1 / n}+e / \varepsilon . \quad$ Здесь $e=E / E_{c}, \quad i=J /\left(\eta J_{0}\right), \quad \theta=$ $=T / T_{c}, \varepsilon=\eta J_{0} \rho_{m} /\left((1-\eta) E_{c}\right), j_{c}(\theta)=(1-\theta)^{\gamma} \beta_{1}$. Подстановка $u=1-e /(i \varepsilon)$ приведет к уравнению $j_{c}(0)=$ $=\frac{i}{(i \varepsilon)^{1 / n}} \frac{u}{(1-u)^{1 / n}}$.

Так как $u \ll 1$, то, раскладывая в степенной ряд сомножитель $(1-u)^{-1 / n}$, получим

$$
j_{c}(\theta)=\frac{i}{\left(i \varepsilon_{1}\right)^{1 / n}}\left(u+\frac{u^{2}}{n}+\frac{n+1}{2 n^{2}} u^{3}+\ldots\right) .
$$

Тогда в линейном по $u$ приближении найдем

$\frac{E}{E_{c}}=i \varepsilon\left[1-\frac{(i \varepsilon)^{1 / n}}{i} j_{c}(\theta)\right]=i \varepsilon\left[1-\frac{(i \varepsilon)^{1 / n}}{i}(1-\theta)^{\gamma} \beta_{1}\right]$.
Используя разложение по степеням $\theta$, будем иметь

$$
\frac{E}{E_{c}}=i \varepsilon\left[1-\frac{(i \varepsilon)^{1 / n}}{i} \beta_{1}(1-\gamma \theta+\ldots)\right] .
$$

Учет нелинейных членов разложения по $u$ позволяет оценить изменение зависимости $E(T)$ более точно. В частности, квадратичное приближение может быть записано в виде

$$
\frac{E}{E_{c}}=i \varepsilon\left[1-\frac{(i \varepsilon)^{1 / n}}{i} j_{c}+\frac{(i \varepsilon)^{2 / n}}{m^{2}} J_{c}^{2}\right] .
$$

Следовательно, при $T_{0}<T \leq T_{c B}$ по мере увеличения температуры технического сверхпроводника увеличение значений $E(T)$ будет происходить практически пропорционально уменьшению его критической плотности тока сверхпроводника, а именно

$$
E(T) \approx\left[J-\left(\frac{J \rho_{m}}{(1-\eta) E_{c}}\right)^{1 / n} \eta J_{c}(T)\right] \frac{\rho_{m}}{(1-\eta)} .
$$

Если $J_{c}$ линейно уменьшается с температурой, то напряженность электрического поля будет нарастать практически линейно. Кроме этого, как следует из (8) и демонстрирует рис. 8, линейный характер увеличения с температурой зависимости $E(T)$ соблюдается тем лучше, чем выше показатель нарастания ВАХ. В предельном случае $n \rightarrow \infty$ формула (9) описывает значения $E(T)$, которые совпадают со значениями, следующими из модели с идеальной ВАХ.

Принимая во внимание (1) и (9), нетрудно найти изменение с температурой плотности тока в сверхпроводнике. Соответствующее выражение может быть записано в виде

$$
J_{s}(T)=\left(\frac{J}{(1-\eta) J_{m}} \frac{E}{E_{c}}\right)^{1 / n} J_{c}(T) .
$$

Данная формула обосновывает результаты, приведенные на рис. 9. А именно ток в сверхпроводнике с реальной ВАХ всегда больше его критического тока, за исключением предельного случая $n \rightarrow \infty$, когда $J_{s} \rightarrow J_{c}$ в силу соблюдения предельного перехода к модели с идеальной BAX.

При $T>T_{c B}$ ток в рамках модели с идеальной ВАХ течет только в стабилизирующей матрице. Поэтому зависимость $E(T)$ будет изменяться в соответствии с температурной зависимостью $\rho_{m}(T)$. Аналогичный вывод, а именно $E \sim J \rho_{m} /(1-\eta)$ следует из (9), если выполняется условие

$$
J \gg\left(\frac{J \rho_{m}}{(1-\eta) E_{c}}\right)^{1 / n} \eta J_{c}(T) .
$$

Оно позволяет оценить температуру технического сверхпроводника со степенной ВАХ, после превышения которой основная часть тока будет течь по матрице. 
Таким образом, джоулево тепловыделение в технических сверхпроводниках с реальной ВАХ при увеличении их температуры изменяется следующим образом. При небольших перегревах $\left(T \ll T_{c B}\right)$, когда основная часть тока течет по сверхпроводнику, диссипируемая энергия экспоненциально нарастает с температурой. По мере ее увеличения транспортный ток постепенно уходит в несверхпроводящую матрицу (рис. 9), и зависимость $G(T)$ начинает нарастать с температурой по закону, практически соблюдающему пропорциональное уменьшение с температурой критической плотности тока сверхпроводника. Данная закономерность обусловлена стабилизирующим действием несверхпроводящей матрицы, которая позволяет избежать пережога технических сверхпроводников в диапазоне температур вплоть до $T_{c B}$. При дальнейшем увеличении температуры $\left(T>T_{c B}\right)$ характер изменения $G(T)$ в основном зависит от температурной зависимости удельного электрического сопротивления стабилизирующей матрицы. При высоких перегревах зависимость $\rho_{m}(T)$ у технических сверхпроводников, покрытых серебром, с хорошей степенью точности может быть описана линейной зависимостью. Поэтому и зависимость $G(T)$ увеличивается с температурой практически линейно.

\section{Выводы}

Условия тепловой стабилизации интенсивно охлаждаемого технического сверхпроводника изучены с учетом нелинейных температурных зависимостей критической плотности тока сверхпроводника, удельного электрического сопротивления стабилизирующей матрицы и теплового потока в хладагент. Проведенный анализ основывался на использовании модели сверхпроводника с идеальной вольт-амперной характеристикой, предполагающей скачкообразный переход из сверхпроводящего состояния в нормальное, и модели сверхпроводника со степенной вольт-амперной характеристикой. В рамках данных моделей установлено, что у интенсивно охлаждаемых токонесущих элементов сверхпроводящих магнитных систем могут отсутствовать минимальные токи существования и распространения нормальной зоны, определяющие диапазон токов, в котором сверхпроводящее состояние устойчиво к произвольным тепловым возмущениям. Данная особенность условий тепловой стабилизации будет наблюдаться в тех случаях, когда в силу интенсивного увеличения с температурой удельного электрического сопротивления стабилизирующей матрицы нарастание мощности джоулева тепловыделения при температурах выше критической температуры сверхпроводника превышает температурный рост мощности теплового потока в хладагент.

Показано, что предельно допустимые токи у сверхпроводников с реальной вольт-амперной характеристикой, охлаждаемых жидкими гелием или водородом, следуют из условия срыва режима пузырькового кипения. В результате, при охлаждении технических сверхпроводников жидкими гелием или водородом устойчивые значения токов в несколько раз превышают условно заданное значение критического тока.

Выполнен анализ взаимосвязанного нарастания с температурой джоулева тепловыделения и теплового потока в хладагент. Показано, что в области возникновения неустойчивости изменение теплового состояния даже у интенсивно охлаждаемых технических сверхпроводников может происходить при условиях, близких к адиабатическим. Эта закономерность определяет особенности условий возникновения пережога технического сверхпроводника при его охлаждении жидким хладагентом. При этом с увеличением критической плотности тока сверхпроводника вероятность его пережога возрастает.

Формирование тепловых состояний технических сверхпроводников с реальными вольт-амперными характеристиками является прямым следствием закономерностей развития в них диссипативных явлений. Доказано, что при делении тока между сверхпроводником и стабилизирующей матрицей ток в сверхпроводнике с реальной вольт-амперной характеристикой всегда больше его априори заданного критического тока. В то же время модель сверхпроводника с идеальной вольт-амперной характеристикой при температурах, превышающих так называемую температуру резистивного перехода, приводит к завышенным расчетным значениям токов, протекающих в стабилизирующей матрице. Поэтому джоулевы потери энергии, вычисленные в рамках модели с непрерывно настающей вольт-амперной характеристикой, в широком диапазоне изменения температуры технического сверхпроводника всегда меньше соответствующих значений, определенных согласно модели с идеальной вольт-амперной характеристикой.

\section{Список литературы}

[1] Альтов В.А., Зенкевич В.Б., Кремлев М.Г., Сычев В.В. Стабилизация сверхпроводящих магнитных систем. М.: Энергоатомиздат, 1984. 312 с.

[2] Уилсон М. Сверхпроводящие магниты. М.: Мир, 1985. $407 \mathrm{c}$.

[3] Гуревич А.Вл., Мини Р.Г., Рахманов А.Л. Физика композитных сверхпроводников. М.: Наука, 1987. 240 с.

[4] Romanovskii V.R., Watanabe K., Awaji S., Nishijima G. // Superconductor: Science and Technology. 2006. Vol. 19. N 8. P. 703-710.

[5] Brentari E.G., Smith R. // Adv. Cryo. Engn. 1965. Vol. 10. P. 325-341.

[6] Bottura L. Critical Surface for BSCCO-2212 Superconductor, Note-CRYO/02/027, CryoSoft library, CERN, 2002.

[7] Dresner L. // Cryogenics. 1993. Vol. 33. N 9. P. 900-909.

[8] Lim H., Iwasa Y. // Cryogenics. 1997. Vol. 37. N 12. P. 789-799.

[9] Keilin V.E., Romanovsky V.R. // Cryogenics. 1982. Vol. 22. N 6. P. 313-317.

[10] Maddok B.I., James G.B., Norris W.T. // Cryogenics. 1969. Vol. 9. N 8. P. 261-273. 